nent. If the poison were absorbed and acted on the centre of the motor nerves of the eye, both eyeballs would be equally affected; but, on the contrary, as we have said, the poisoned eye always bulges more than the other, whence we conclude that it is probable gelseminum poisons the terminations of the motor nerves of the eye, though the centre of these nerves may be also affected.

We tried to ascertain if the nerves of the poisoned eye were more depressed than those of its fellow. Immediately after death we opened the skull, removed the brain, exposed the third nerve leading to each orbit, and applied electricity. Each nerve, so far as we could ascertain, conducted the current; hence the paralysis was far from complete, and there seemed no marked difference between their conductibility. This experiment is very difficult to manage, and is not very trustworthy ; for the nerves of warm-blooded animals, after death, soon cease to conduct impressions, and they are quickly exhausted by electricity. Moreover, we should not expect to find any decided, or perhaps any detectable, difference between the two nerves, since both were paralysed, both eyes being prominent, though the poisoned eye bulged most. Again, as sufficient poison was taken up to paralyse the respiratory centre, enough was likewise absorbed to paralyse the nerves of the opposite eye; hence we could hardly expect any obvious difference in the conductibility of the two nerves.

The interesting effects from the local application to the rabbit's eye led us (skilfully helped by Mr. Pearse on this as on many other occasions) to try the drug on two men. We dropped into the eye of each patient two drops of the alkaloid solution every hour for eight hours. The pupils in an hour and a half became very widely dilated. After the fourth application, one man complained of slight frontal headache and some giddiness, subsequent doses being fol lowed by slight temporary frontal headache. In spite of this prolonged action we failed to produce any decided prominence of the poisoned eye. Several who saw the man thonght the trial eye was a little the more prominent, but we were at the best doubtful on this point. We produced no paralysis of the muscles, as shown by the absence of diplopia, and the poisoned eye moved in every direction as well as its fellow. The man suffered from a violent cough, and during a paroxysm his face became much congested; yet even then the poisoned eye did not protrude. Thus we did not get any topical action on the terminations of the third nerve, though in each case the absorption of the poison was made manifest by the widely dilated pupil, and the giddiness and headache which seized the patient.

These experiments prove that gelseminum partially paralyses the nerves of the orbits, affecting the terminations before the trunks of the nerves; and our observations on man further show that it paralyses the sixth nerve first, for the external rectus is weakened before the other muscles, and that afterwards it affects the third nerve, for the inward, upward, and downward movements of the eye are impaired, and ptosis is often marked.

$$
\text { ( } T o \text { be continued.) }
$$

\section{ON BORACIC ACID AS AN ORDINARY DRESSING FOR WOUNDS.}

BY LEONARD CANE, M.D., B.S. LOND.,

CONSULTING SURGBON TO THE PBTEBBOROUGH GENBRAL IAFIRMARY.

THE use of boracic acid and the different preparations containing it has been introduced as part of the antiseptic system, and its advantages have been well dwelt upon by Professor Lister, Mr. Godlee, and others (see THE LANCET, May 17th and 24th, 1873). But it is as a simple dressing for wounds of all kinds, altogether apart from the antiseptic system, strictly so called, that I wish to draw attention to it.

However great the advantages of Mr. Lister's method of dressing wounds, it is undoubtedly felt by the great majority of surgeons, especially those engaged in private practice and whose time is of ten limited, that the details and the time required for their proper performance, practically prevent its use in all ordinary cases. In hospital practice, where skilled assistance is always at hand, and in the higher class of purely surgical practice, I believe that the anti- septic mode of dressing wounds is by far the best. But we want something which, while it has to a certain extent the merits, yet is without those tedious details, and which can readily be performed by anyone without assistance.

The preparations of boracic acid have now been rather extensively tried by me for some months, and in all the cases in which they have been used the results have been good, and decidedly better than under the ordinary methods of dressing. The most convenient forms for use are the boracic (boric) lint and cotton wool, a concentrated watery solution of the acid, and boracic ointment. Boracic lint is prepared by soaking lint in a saturated boiling solution of the acid. On drying the lint a copious deposit of fine flaky crystals takes place between its fibres. Cotton wool may be similarly served, and when dried and carefully picked out forms a very useful dressing. The concentrated solution is made by dissolving the acid in boiling water to saturation. The ointment is made by rubbing down one drachm of the acid with one ounce of simple ointment, or benzoated lard.

Boracic acid, unlike most antiseptic agents, is bland and unirritating; and, whilst its non-volatility renders it less useful in some cases than carbolic acid, its great superiority to this and to chloride of zinc resides in its unirritating nature. The boracic lint is best used as a dry dressing, and for recent wounds where simplicity is desired it has no equal. A pad of lint applied immediately over the wound, and kept in place by pieces of strapping, is all that is required, and union by first intention is a common result.

The following cases will illustrate its application to this kind of wound.

An elderly gentleman was drawing the cork of a wine bottle and applying considerable force when the shoulder of the bottle broke in his hand, and he received a jagged wound obliquely across the palm of the left band, extending from the ulnar side of the wrist to the cleft between the thumb and forefinger. Considerable bleeding took place and several small arteries were wounded, but the hæmorrhage was temporarily stopped by tightly binding up the hand and wrist. The wound was gently bathed with some boracic lotion and brought together by three silver-wire sutures. A long pad of boracic lint, about four folds thick, was then placed over the wound and firmly strapped down by plaster. The whole hand was then firmly bandaged. On the third day the pad was removed. There had been some bleeding; there was very little swelling of the hand, and no offensive smell to be detected. On taking off the pad the wound was seen to have united at its extremities, where it was more cleanly cut, and at the deepest and most irregular part it was looking healthy. There was a little suppuration, but no odour of putrefaction. The stitches were taken out and the hand dressed as before. On the sixth day the dressings were again undone, and the wound was then found to have entirely healed except about half an inch of its length, which had become superficial and was covered with a whitish glairy discharge; there was no fetor or evident suppuration. The dressings were reapplied, and on the ninth day were re moved altogether, the wound having completely healed, leaving a firm cicatrix. This case illustrates the simplicity of the method, for with a severe wound, four inches long, nothing further was required than three simple dressings.

J. B $\longrightarrow$, aged twenty, labourer, was knocked down by the wheel of a thrashing machine and received a severe wound on the outer side of the left knee, part of the wheel passing over the side of the knee. On examination I found a contused wound, the surface of which was almost reduced to a pulp, and measuring about two inches across in any direction, whilst in the centre it was half an inch deep. There was some swelling of the knee-joint, which had come on very rapidly, but beyond this no injury to the joint itself could be detected. The trousers over the knee had been torn, and the wound was very dirty. It was not bleeding to any extent. I first washed out the wound with some boracic lotion, pouring it form a height in a gentle stream, so as to remove the particles of dirt, \&c., then applied a pad of boracic lint several folds thick, and placing a splint bebind the knee to keep it at rest, firmly fixed the whole with a bandage. On the following day the dressings were removed. There had been a great amount of discharge, and the dressings were soaked with it. The discharge consisted of pus and débris from the wound, and was decidedly putrefactive. There was considerable inflammation round the wound, and the knee was swollen and so painful that the 
patient could not sleep. The wound was well washed out with boracic lotion, and the pad applied as before. Dressings taken off next day; swelling, pain, and redness less marked; discharge still profuse, but no longer fetid. Dressed as before. For the next three days the wound was dressed daily and made steady progress; after this at intervals of three days for a fortnight, during which it granulated and sicatrised, forming a good cicatrix. For the latter part of the time the patient dressed the wound himself, simply applying a piece of boracic lint.

In cases of phlegmonous erysipelas, where sloughing takes place, the boracic lotion appears to have a very beneficial effect, and to stop further spread of the mischief. The following case illustrates its use.

A. M- - aged fifty-six, female, who had suffered from ulcers on the leg some years previously, was taken suddenly ill with rigors, vomiting, and fever, without any apparent cause beyond "catching cold." She lived in the country; had not been where there was any illness, and had not received any wound or injury. She took to her bed at once, and complained of severe pain on the outer side of the left instep and up the calf of the leg. In the course of a few hours the leg swelled to a great extent, and was intensely painful. Patient became delirious, and her temperature and pulse ran up quickly. The leg soon became brilliantly red and extremely painful and tender. On the third day the swelling about the foot felt " boggy," but there was no fluctuation to indicate any circumscribed collection of matter. Large blebs formed on the surface of the instep and ankle. The next day a patch, five inches long by two and a half inches broad, appeared on the outer side of the foot, paler than the surrounding skin, of a yellowish marbled colour, and evidently about to slough. No deep fluctuation could be found anywhere, and it was not thought advisable to make any incisions. The skin over the patch gave way on the following day, and the sloughing process appeared to be rapidly extending. The whole leg was very much inflamed and swollen, and red lines, following the course of the lymphatics, were seen on the thigh. Up to the present time the chief treatment had been fomentations to the leg, while nourishment in every available shape was given to the woman. The erysipelatous state appearing to spread, and a large slough having made its appearance on the inner side of the foot just below the ankle, the whole leg and foot were ordered to be bathed at frequent intervals with a warm solution of boracic acid, and the foot to be kept constantly wrapped in linen eloths wetted with the same. Liberal support, with quinine and perchloride of iron, to be continued as before. The next day there was some improvement: the inflamed lymphatics upon the thigh were less tender; the redness of the foot and leg had somewhat diminished; the sloughing had not extended. Dressed as before. In the course of the next two days the swelling of the leg and foot decreased; the large slough on the outer side of the foot became surrounded by a line of demarcation and began to separate. No extension of the disease had taken place, and the patient had decidedly improved in her general symptoms. The sloughs separated in the course of a week, leaving a large surface about one-third of an inch deep, which suppurated freely, but was without any odour of putrefaction. Patient expressed herself as greatly relieved by the lotion, and said that during the night, if not dressed for a few hours, it became much more painful. From this time the large wounds gradually, and without any interruption, healed. The granulations were healthy and the discharge comparatively slight. The whole of both wounds cicatrised well, and patient entirely recovered the use of her limb, though with some stiffness.

In this case the effect of the antiseptic on the spread of the erysipelatous inflammation was very evident, and the whole progress of the case fully bore out the advantages which were expected from the boracic acid.

As a simple dressing for old ulcers of the leg the boracic lotion and lint are cleanly, and the healing processes go on favourably under its use, sometimes succeeding when other applications have failed.

J. G-, aged thirty-six, had large deep ulcers which followed her confinement two years ago. They were situated on the inner side of the ankle and below the knee. She was ordered to rest her leg up, and was given liberal diet; whilst the wounds were dressed with sulphate-of-zinc lotion (" red wash") for some weeks. Under this treatment they improved slowly, but not very satisfactorily. The dressings became fetid occasionally from neglect, and for some weeks the ulcers got better and worse alternately. The ulcers were accordingly well washed with boracic lotion every morning, and afterwards covered with boracic lint. Under this treat ment a marked change occurred; the ulcers became clean, lost all their fetor, and were completely healed in three weeks' time.

In skin-grafting the boracic acid is very useful. I have found the following plan answer best:- The surface to which it is intended to apply the grafts is first well cleaned by applying the boracic lotion for some days, till the discharge is nerfectly free from putrefactive odour. The skin from which it is intended to take the grafts is also well washed with the lotion. Small portions of the skin are then removed, and applied to the raw surface in the usual way. I then protect the grafts by a strip of gntta-percha tissue dipped in the boracic lotion, and over all apply a piece of boracic lint. Under this plan the grafting is very successful, and gives very little trouble. The following case will illustrate the method:-

A. $\mathrm{P}-$, aged thirty-six, domestic servant, applied on account of a large varicose ulcer on the leg. The ulcer was of old standing, and had broken out and healed over several times before. When first seen it measured about two inches in all directions. It had raised and indurated edges. The floor of the ulcer was dark purple, and the granulations were unhealthy. The skin round the ulcer was dark and congested. Patient had worn a bandage for some time, and could apply it evenly. The ulcer was first well washed with boracic lotion, covered with boracic lint, and then the leg was well strapped from the ankle to above the ulcer. Patient was given some boracic lotion, with orders to remove the strapping after three days, and then apply linen rags twice a day wet with the lotion. At the expiration of a fortnight she came again. The wound was much healthier in appearance. The edges were reduced and less prominent. The granulations were redder, and the discharge less, and quite inodorous. Five grafts were now applied to the ulcer, the skin being taken from the woman's arm, and the whole dressed as described above. When nex seen (ten days afterwards) the wound had lessened in size to the diameter of an inch. Three of the grafts had grown considerably, and one had joined the edge of the ulcer. The wound looked healthy, though patient had been obliged to walk about considerably. There was now very little pain. She continued to apply the lotion once a day, and the wound completely healed in a short time.

This case shows how little trouble the dressing gives. After the grafts were applied the patient had to walk away from my residence. She was unable to rest up, and could only apply the lotion once a day. Yet with this little attention it made steady progress, and patient was more free from pain than she had been for months.

For boils on the neck and elsewhere the boracic lint is an excellent application; a piece large enough to hide the boil, and covered with a piece of gutta-percha tissue, of ten gives great relief. For carbuncles and other cases in which it is desired to apply a poultice, I have found the new "instantaneous poultice," prepared from Iceland moss by Messrs. Rigollot, a capital and efficient remedy. The poultice should be prepared by soaking it for a short time in the boracic lotion, and when applied should be covered with gutta-percha tissue.

Lastly, in some of the vegetable parasitic diseases, such as pityriasis versicolor, tinea circinata, \&c., the boracic lotion and vintment will of ten be found serviceable.

Briefly to sum up the advantages of boracic acid:-

1. It is an antiseptic which does not irritate and inflame, and so allows the natural processes of healing to go on without much interruption.

2. It is exceedingly simple in its application, and can be used apart from all the details required by a thoroughly antiseptic method.

3 . It can be used in the shape of the lint, lotion, cottonwool, \&c., in combination with most other methods of treatment.

4. Its cost is trifing; and though this is of secondary importance, it is a feature of the treatment which will recommend its employment in workhouse infirmaries and in dispensary and parish practice.

Peterborough. 